\section{The influence of Mediterranean diet in acne pathogenesis and the correlation with insulin-like growth factor-1 serum levels: Implications and results}

\author{
Mariabeatrice Bertolani, ${ }^{1}$ \\ Eleonora Rodighiero, ${ }^{1}$ Roberta Saleri, ${ }^{2}$ \\ Giuseppe Pedrazzi, ${ }^{3}$ Simona Bertoli, ${ }^{4,5}$ \\ Alessandro Leone, ${ }^{4}$ Claudio Feliciani, ${ }^{1}$ \\ Torello Lotti, ${ }^{6,7}$ Francesca Satolli ${ }^{1,8}$
}

${ }^{1}$ Section of Dermatology, Department of Medicine and Surgery, University of Parma; ${ }^{2}$ Department of Veterinary

Science, University of Parma;

${ }^{3}$ Department of Medicine and Surgery, Unit of Neuroscience and

Interdepartmental Centre of Robust Statistics, University of Parma;

${ }^{4}$ International Center for the Assessment of Nutritional Status, Department of Food, Environmental and Nutritional Sciences (DeFENS), University of Milan; 5Istituto Auxologico Italiano, IRCCS, Lab of Nutrition and Obesity Research, Milan; 'University Unit of Dermatology and Regenerative Medicine, University of Rome G. Marconi, Italy; ${ }^{7}$ Department of Dermatology and Communicable Diseases, Ministry of Health, Moscow Russia First Medical Moscow State University, Higher Education Institution I. M. Sechenov, Russian Federation; ${ }^{8}$ Dermatologic Unit, University Hospital of Parma, Italy

\footnotetext{
Abstract

Acne is a chronic inflammatory disease of the pilosebaceous unit, and its etiology is complex and multifactorial. The role of the diet in its pathogenesis is still debated. The purpose of this study was to assess the association between MD and IGF-1 in acne patients and, as secondary objective, the role of systemic treatment on IGF-1 serum levels, in accordance with the patients' diet. This study included 35 patients aged 14-30 years affected by acne and treated in line with the EDF guidelines. Patients were divided into 2 groups based on a questionnaire score assessing the adherence to the Mediterranean diet: the Mediterranean Group (score $\geq 6$ ) and the Western Group $($ score $<5)$. IGF-1 serum levels were measured in all patients before and after treatment and then compared to healthy population. IGF-1 levels were higher in patients
}

than in controls and in the Western group than in the Mediterranean group. We speculate that the Mediterranean diet can have a protective role in the pathogenesis of acne by acting on the systemic route of IGF-1.

\section{Introduction}

Acne vulgaris is the most common chronic inflammatory disease of the pilosebaceus unit. It affects almost all young adults aged 15-17; although its prevalence tends to decrease with age, $15-20 \%$ of adults are affected resulting in negative effects on their self-esteem, mood and quality of life. ${ }^{1}$ Its etiology appears to be complex and multifactorial, involving abnormal follicular keratinization, hyperseborrhoea, Cutibacterium Acnes proliferation in the pilosebaceous unit and inflammatory mediators released into the skin. The role of diet in the literature remains controversial but recent studies have supported the relationship between specific dietary factors and acne. ${ }^{2}$ In particular, an association with dairy products and with high-glycaemicindex foods has been demonstrated. ${ }^{3}$

Diet based on products with a high glycemic index leads to hyperinsulinemia affecting the level of circulating Insulin Like Growth Factor-1 (IGF-1), which has been proposed to have a key role in acne pathogenesis and acne has been proposed to be an IGF-1-mediated disease. ${ }^{3}$ The regulation of IGF-1 by diet is complex, however, several previous randomized controlled trials found that a Mediterranean diet (MD) may reduce the biological activity of IGF1., ${ }^{4,5}$ The typical MD pattern is characterized by low-glycaemic-index foods, low amount of dairy products, high amount of antioxidant and antinflammatory nutrients, that may be able to modulate IGF-1 plasma concentration. Moreover, given the existing evidence on acne and inflammatory pathways, the antioxidant and anti-inflammatory effects of MD may be protective against the onset of diseases. However, no previous observational or prospective cohort study has been conducted to assess the role of MD on acne in relation to IGF-1 plasma concentration modulation and on the effect of drugs treatment on IGF-1 plasma concentration according to baseline dietary pattern. ${ }^{6}$ The purpose of this study was to assess the association between MD and IGF-1 in acne patients and, as secondary objective, the role of systemic treatment on IGF-1 serum levels, in accordance with the patients' diet.
Correspondence: Eleonora Rodighiero, Dermatology Unit, Department of Medicine and Surgery, University of Parma, Via Gramsci 14, 43126 Parma, Italy.

Tel.: +39-0521-033562 - Fax: +39-0521702959.

E-mail: eleonora.rodighiero@gmail.com

Key words: Acne; Insulin Like Growth Factor-1; IGF-1; Mediterranean diet.

Acknowledgements: The authors would like to thank Rosalia Aloe.

Contributions: MB: conceived and designed the analysis, collected the data and wrote the paper. ER: conceived and designed the analysis, collected the data and wrote the paper. RS: contributed data and analysis tools. GP: performed the analysis. SB: contribuited to the analysis and interpretation of data of the work. AL: contributed to the analysis and interpretation of data of the work. CF: conceived and designed the analysis. TL: conceived and designed the analysis. FS: conceived and designed the analysis, collected the data and wrote the paper.

Conflict of interest: The authors declare that they have no conflict of interest.

Funding: None.

Availability of data and material: Data and materials are available by the authors.

Informed consent: Obtained.

Please cite this article as: Bertolani $M$, Rodighiero E, Saleri R, et al. The influence of Mediterranean diet in acne pathogenesis and the correlation with insulin-like growth factor1 serum levels: implications and results. Dermatol Rep 2022;14:9143.

Received for publication: 18 March 2021. Accepted for publication: 29 November 2021.

This work is licensed under a Creative Commons Attribution-NonCommercial 4.0 International License (CC BY-NC 4.0).

OCopyright: the Author(s), 2022

Licensee PAGEPress, Italy

Dermatology Reports 2022; 14:9143

doi:10.4081/dr.2022.9143

\section{Materials and methods}

This study included 35 participants with acne vulgaris aged 14-30 years who were affected by mild, moderate, and severe acne. Adherence to the traditional MD was assessed using a validated 14-item questionnaire ${ }^{6}$. Values of 0 or 1 were assigned to each of 14 dietary components. The ques- 
tionnaire was considered valid only when all 14 items were completed. Participants who scored $\geq 6$ points were considered compliant with the MD and were defined Mediterranean Diet Group (MD-Group), who scored $<5$ points were considered compliant with Western Diet and formed the Western Diet Group (WD-Group). The control group was composed of 15 agematched subjects without clinically manifest acne or its consequences at the time of the study.

The subjects' age, gender, body mass index (BMI), waist circumference and severity of acne were recorded. Patients with endocrinological pathologies, those who were underweight, overweight, or subjects with other diseases or on medication that could interfere with IGF-1 were excluded from the study.

A clinical diagnosis of acne vulgaris was made after examining the patients in a proper daylight-illuminated examination room by an experienced dermatologist. Scoring of the severity of acne vulgaris was evaluated by the same dermatologist with the Global Acne Grading System (GAGS). ${ }^{7}$

The GAGS score range of our patients was between 1 and 38 , so we considered three groups. Every group received a treatment chosen according to the recent clinical European Dermatology Forum guidelines and to acne severity. Specifically, patients with mild acne (GAGS $\leq 18$ ) received Clindamycin $10 \mathrm{mg} / \mathrm{g}+$ tretinoin $0.25 \mathrm{~g}$ gel for 3 months; the ones with moderate acne (GAGS 19-30) received oral lymecycline $300 \mathrm{mg}+$ topical benzoyl peroxide $5 \%$ gel for 3 months; the ones with severe acne (GAGS $\geq 31$ ) received Isotretinoin $0,5 \mathrm{mg} / \mathrm{kg} /$ die for 8 months (total cumulative dose of $120 \mathrm{mg} / \mathrm{kg}$ ). The clinical evaluation relies on 3 medical examinations: T0 (first visit), T1 (1 month treatment) and T2 (end of treatment). For patients with severe acne the follow up was every 2 months for 8 months. During each examination, the GAGS score has been calculated, possible side-effects has been investigated and any change in patients' life quality documented.

Blood was drawn from each fasting participant during T0 and during T2 (after 3 months for patients with mild and moderate acne and after 8 months for patients with severe acne). Blood samples were drawn by venipuncture and centrifuged by a trained phlebotomist; the serum was separated and stored at $-25^{\circ} \mathrm{C}$. In order to avoid variation, all samples were studied on the same day and using the same kit. IGF-1 levels were determined by an IDS-iSYS automated IGF-I immunoassay (IDS-iSYS Insulin-like Growth Factor-I, Immunodiagnostic Systems Limited, Boldon, Tyne \& Wear,
NE35 9PD, UK). The study was conducted according to the rules of good clinical practice and the study protocol was approved by the local ethics committee. Eligible participants were recruited at the Dermatology Clinic of Parma between Dec 2018 and Dec 2019. Before enrollment, written consent was obtained from each participant or guardian (if the participant was aged $<18$ years).

\section{Results}

We analyzed 35 patients: 25 female and 10 males with a mean age of $21.02 \pm 4.8$ years; 27 patients belong to the MD group and 8 belong to the WD Group. Data were compared to a control group including 7 males and 6 female subjects, with a mean age of $26.60 \pm 2.16$ years. Serum IGF-1 levels were within the normal reference range. There is a statistically significant difference $(\mathrm{P}=0.05)$ in serum IGF-1 levels between controls and patients, with higher values in the patient group.

At $\mathrm{T} 0$ the medium value of IGF-1 serum levels of the MD group was 270,9 $\mathrm{ng} / \mathrm{mL}$ and for the WD group was 350,5 $\mathrm{ng} / \mathrm{mL}$ : the difference between the two groups is statistically significant $(\mathrm{P}=0.027)$.

There is a statistically significant decrease of IGF-1 level for the patients belonging to the WD group after two months of acne therapy. Conversely, the patients of the MD group have a lower serum IGF-1 value at T0 with no variation

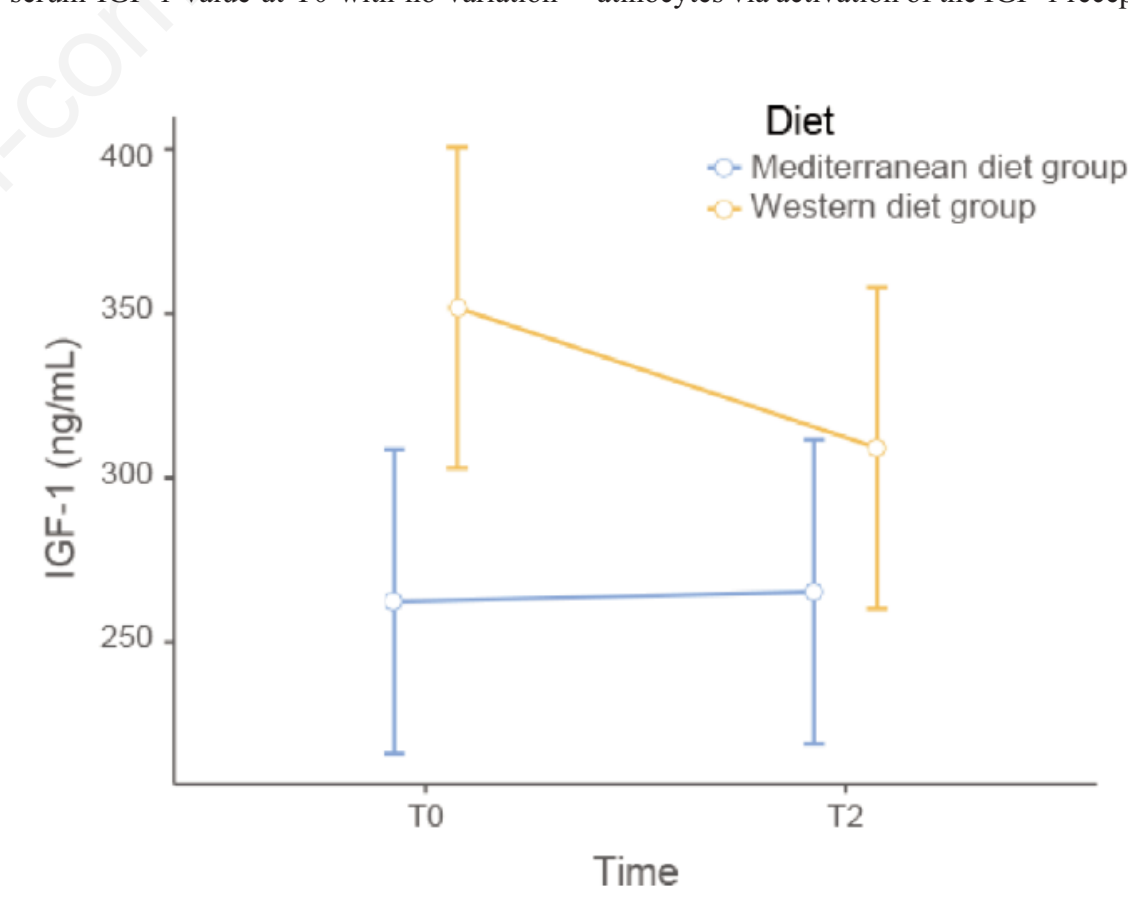

Figure 1. Mean values and 95\% confidence interval (95\%-CI) of IGF-1 levels stratified by the type of diet at T0 (first visit) and T2 (end of acne treatment). after two months of acne therapy (Figure 1)

Similar results were obtained when we considered only the patients with severe acne, who were treated with isotretinoin. Specifically, there is a statistically significant difference in IGF-1 serum levels between the WD group and the MD group at $\mathrm{T} 0$. In addition, for the first group there is a reduction of IGF-1 serum levels after six months of therapy.

In patients with mild and moderate acne, there is no statistically significant reduction of IGF-1 serum levels after topical or systemic antibiotic treatment in both groups (Figure 2)

Acne severity at baseline differed between MD and WD patients even if there is not a statistically significant difference due to the small sample size (Figure 3).

\section{Discussion and conclusions}

Although the past dermatological doctrine denied a direct involvement of diet in acne pathogenesis, the most recent research has shown a relationship between excessive intake of dairy products, hyperglycaemic food and acne. ${ }^{8}$ Current knowledge supports the evidence that a Western diet induces hyperinsulinemia and subsequently raises IGF-1 serum levels which may play a crucial role in the pathophysiology of acne. In fact, IGF-1 stimulates sebocyte growth, lipogenesis of sebaceous glands, androgen synthesis and promotes proliferation of keratinocytes via activation of the IGF-1 recep- 
tor. Karadag et al. ${ }^{9}$ in 2010 assumed and demonstrated that IGF-1 levels decreased after short-term isotretinoin treatment. Recently, Rodighiero et al. have confirmed that common therapies for acne are able to modulate the systemic route of IGF-1, but not to reduce it with statistical significance. ${ }^{10}$

Circulating levels of IGF-1 are used to evaluate the bioactivity of IGF-1 on tissue because of the lack of alternative techniques in vivo. Its bioactivity depends not only on IGF-1 serum levels but also on IGF binding protein levels, on genetic factors and on local production. Even though different elements such as diet, age, sex or hormones can influence IGF-1 levels, more than $60 \%$ is genetically determined. Hence it has been suggested by Tasli et al. that the only way to adjust IGF-1 levels is to act on the unique manageable factor, represented by diet. ${ }^{11}$ Furthermore, several previous randomized controlled trials found that a MD may reduce the biological activity of IGF-1. ${ }^{5}$

According to Melnik, dietary intervention in acne patients should decrease fat, glucose and total energy intake; furthermore, the diet can diminish insulin/IGF-1 signaling, which is mainly mediated by milk protein assumption. The same author proposes an increase in fruit and vegetable consumption, reducing animal derived food and recommends a paleolithic diet. ${ }^{12}$

Interestingly, Jung et al have shown a lower incidence of acne in Koreans adhering to a traditional Korean diet, with a low glycemic index and low-fat content and, conversely, a higher incidence in people following a westernized diet. ${ }^{13}$

Along with these factors we have hypothesized that the MD could be the most suitable diet for our patients. It is characterized by abundant plant foods (fruit, vegetables, bread, other forms of cereals, potatoes, beans, nuts, and seeds), fresh fruit as the typical daily dessert, low to moderate consumption of fish, poultry, eggs and dairy products (predominantly cheese and yogurt) and low amounts of red meat. ${ }^{14}$ The main sources of fat and alcohol are primarily virgin olive oil and wine respectively. They contain hydroxytyrosol and tyrosol, oleocanthal, resveratrol, and many other dietary bioactive phenolic compounds with substantial anti-inflammatory properties. Consequently, the Mediterranean diet is rich in antioxidant compounds and bioactive elements with anti-inflammatory effects and has a low glycemic index. ${ }^{15}$ It is well known that this dietary pattern is healthy and has positive effects on longevity, quality of life and disease prevention such as metabolic syndrome, cardiovascular diseases and could reduce the incidence of cancers. ${ }^{14}$ Concerning acne pathogenesis, the protective role of Mediterranean diet was first assumed and demonstrated by Skroza et al. ${ }^{16}$ and, more recently, confirmed by the studies of Claudel et al. ${ }^{17}$ Latterly, Donnarumma et al. ${ }^{18}$ suggested a possible protective role of resveratrol on acne by investigating adherence to the Mediterranean diet, using the PREDIMED questionnaire, and the severity of acne in patients and controls. Barrea et al. ${ }^{19}$ affirmed that high adherence to the MD could be a marker of inflammation in patients with acne and the PREDIMED score $\leq 9$ predicted the highest GAGS score. They suggested that the body composition and PREDIMED scores might represent possible markers of the severity of acne in a clinical setting.

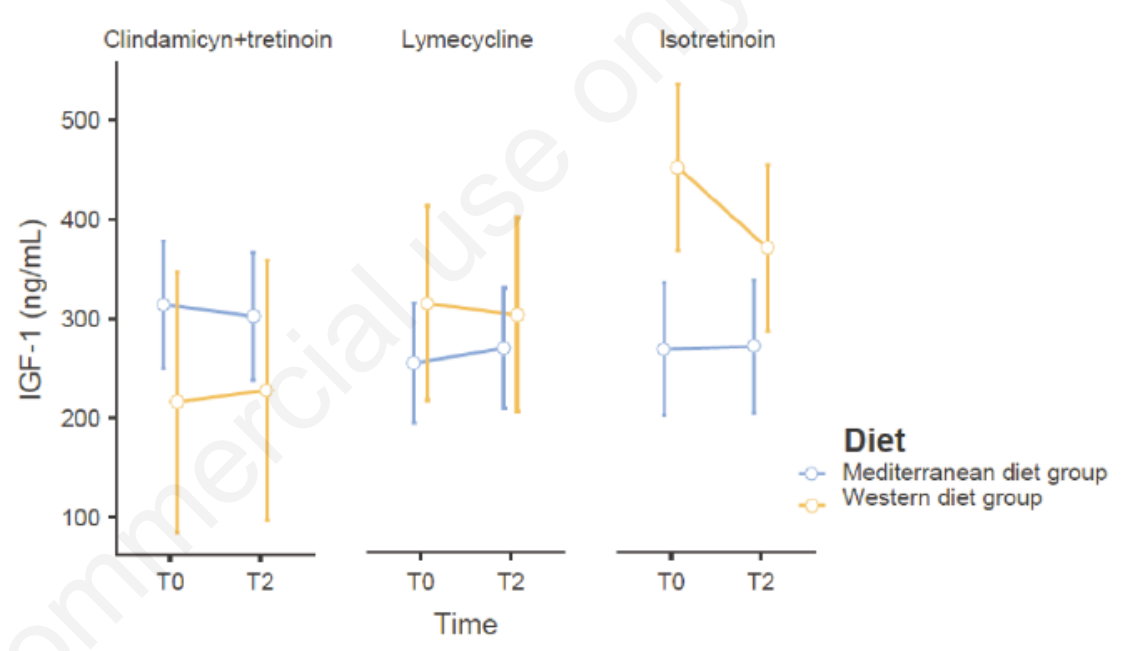

Figure 2. Mean values and 95\% confidence interval (95\%-CI) of IGF-1 levels stratified by the type of treatment and diet at T0 (first visit) and T2 (end of acne treatment).

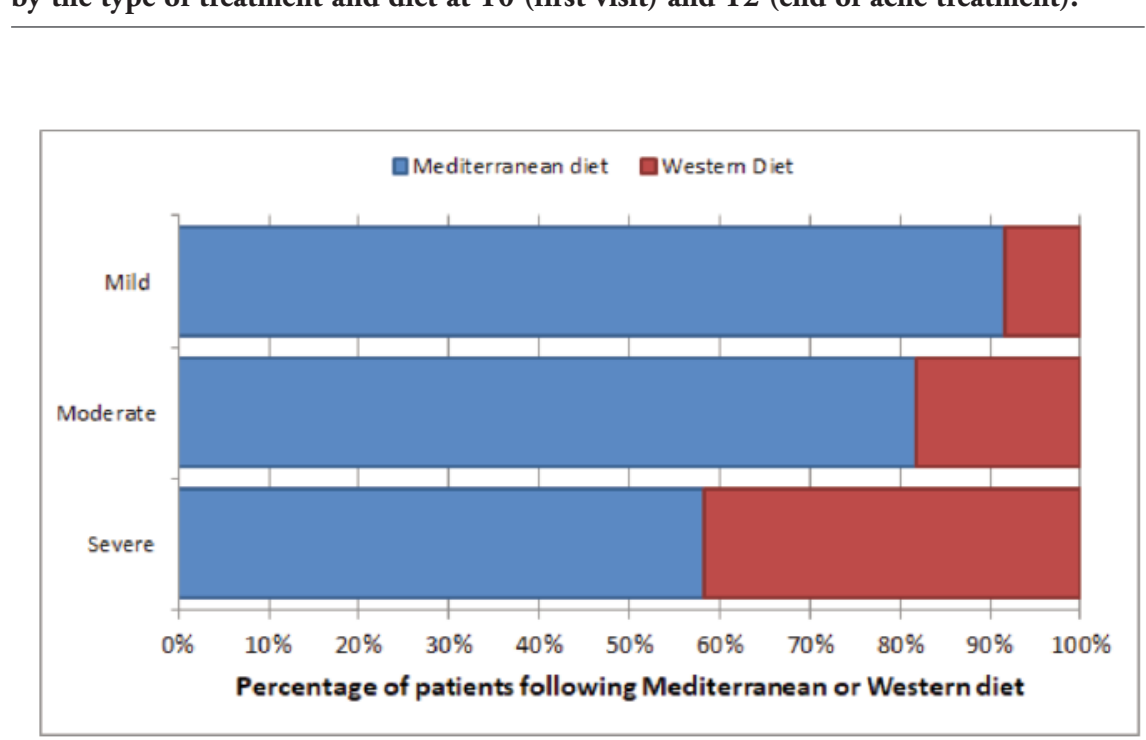

Figure 3. Percentage of patients following Mediterranean diet or Western diet divided

according to acne severity.
In light of this evidence, we have investigated not only adherence to the Mediterranean diet of patients affected by acne vulgaris, but also considered the possible relationship with IGF-1 levels. In our experience, patients with a high-average adherence to the MD exhibited lower IGF-1 serum levels compared to patients that did not follow the MD and this difference is statistically significant (Figure 1). Intriguingly, we found that IGF-1 serum levels of patients with severe acne and low adherence to the Mediterranean diet are reduced after six months of systemic isotretinoin intake (Figure 2), confirming the fact that this therapy is able to modulate the levels of IGF-1 only in patients that do not follow the MD. ${ }^{8}$ Nevertheless we cannot state that this reduction of IGF-1 serum levels after thera- 
py is statistically significant because of the small size of our sample.

Our non-randomized controlled trial supports the influence of a high glycemic diet on the aggravation of acne and the defensive role of the Mediterranean diet suggesting resveratrol and olive oil as protective foods. The promising implications of medical nutrition therapy in acne pathogenesis may be a viable treatment option for patients with acne, due to the diet-induced endocrine effects on acne-promoting pathways. The results of our study may highlight suitable nutrients, such as resveratrol and olive oil that can have a beneficial potential on this disease, hence we consider the Mediterranean diet useful both for Mediterranean and non-Mediterranean populations.

In any case, further clinical and translational research is necessary to investigate and confirm the association between acne and nutrition.

\section{References}

1. Karadağ AS, Balta İ, Saricaoğlu H, et al. The effect of personal, familial, and environmental characteristics on acne vulgaris: a prospective, multicenter, case controlled study. G Ital Dermatol Venereol 2019;154:177-85.

2. Spencer EH, Ferdowsian HR, Barnard ND. Diet and acne: a review of the evidence. Int J Dermatol 2009;48:339-47.

3. Melnik BC, Schmitz G. Role of insulin, insulin-like growth factor-1, hyperglycemic food and milk consumption in the pathogenesis of acne vulgaris. Exp Dermatol 2009;18:833-41.

4. Kaaks R, Bellati C, Venturelli E, et al.
Effects of dietary intervention on IGF-I and IGF-binding proteins, and related alterations in sex steroid metabolism: the Diet and Androgens (DIANA) Randomised Trial. Eur J Clin Nutr 2003;57:1079-88.

5. Tosti V, Bertozzi B, Fontana L. Health Benefits of the Mediterranean Diet: Metabolic and Molecular Mechanisms. J Gerontol A Biol Sci Med Sci 2018;73:318-26.

6. Babio N, Bulló M, Basora J, et al. Adherence to the Mediterranean diet and risk of metabolic syndrome and its components. Nutr Metab Cardiovasc Dis 2009;19:563-70.

7. Doshi A, Zaheer A, Stiller MJ. A comparison of current acne grading systems and proposal of a novel system. Int $\mathrm{J}$ Dermatol 1997;36:416-8.

8. Cordain L, Lindeberg S, Hurtado M, et al. Acne Vulgaris: A Disease of Western Civilization. Arch Dermatol 2002;138:1584-90.

9. Karadag AS, Ertugrul DT, Tutal E, Akin KO. Short-term isotretinoin treatment decreases insulin-like growth factor-1 and insulin-like growth factor binding protein-3 levels: does isotretinoin affect growth hormone physiology? $\mathrm{Br} \mathrm{J}$ Dermatol 2010;162:798-802.

10. Rodighiero E, Bertolani M, Saleri R et al. Do acne treatments affect insulinlike growth factor-1 serum levels? A clinical and laboratory study on patients with acne vulgaris. Dermatol Ther 2020; 13439

11. Tasli L, Turgut S, Kacar N, et al. Insulin-like growth factor-I gene polymorphism in acne vulgaris. J Eur Acad Dermatol Venereol 2013;27:254-7.
12. Melnik B. Dietary intervention in acne: Attenuation of increased mTORC1 signaling promoted by Western diet. Dermatoendocrinol 2012;4:20-32.

13. Jung JY, Yoon MY, Min SU, et al. The influence of dietary patterns on acne vulgaris in Koreans. Eur J Dermatol 2010;20:1-5.

14. Willett WC, Sacks F, Trichopoulou A, et al. Mediterranean diet pyramid: a cultural model for healthy eating. Am J Clin Nutr 1995;61:1402S-6S.

15. Martínez-González MÁ, Hershey MS, Zazpe I, Trichopoulou A. Transferability of the Mediterranean Diet to Non-Mediterranean Countries. What Is and What Is Not the Mediterranean Diet. Nutrients 2017;9: 1226.

16. Skroza N, Tolino E, Semyonov L, et al. Mediterranean diet and familial dysmetabolism as factors influencing the development of acne. Scand J Public Health 2012;40:466-74.

17. Claudel JP, Auffret N, Leccia MT, et al. Acne and nutrition: hypotheses, myths and facts. J Eur Acad Dermatol Venereol 2018;32:1631-7.

18. Donnarumma M, Savastano S, Barrea $\mathrm{L}$, et al. Comment on 'Acne and nutrition: hypotheses, myths and facts'. J Eur Acad Dermatol Venereol 2019;33:e418-9.

19. Barrea L, Donnarumma M, Cacciapuoti $\mathrm{S}$, et al. Phase angle and Mediterranean diet in patients with acne: Two easy tools for assessing the clinical severity of disease. J Transl Med 2021;19:171. 